\title{
Theoretische Grundlagen: Das International Sales Accelerator Modell als Business Development und Vertriebsmanagement Prozess-Tool
}

\subsection{Stand der Business Development- und Vertriebsforschung}

Der Begriff Business Development kann übersetzt werden mit Geschäftsentwicklung bzw. Geschäftsfeldentwicklung. Unter dem Begriff werden unterschiedliche wissenschaftliche Konzepte zusammengefasst (siehe Tab. 2.1). Zum Beispiel verstehen Kohne und Becker darunter hauptsächlich die Entwicklung neuer Geschäftsmodelle ${ }^{1}$. Nach der Definition von Bernecker beinhaltet Business Development die drei Dimensionen Neukundengewinnung, Erschließung neuer Märkte und Entwicklung neuer Geschäftsmodelle ${ }^{2}$. Sorensen versteht darunter die analytische Vorbereitung auf potenzielle Wachstumsmöglichkeiten nicht aber die Implementierung der Wachstumsmöglichkeiten ${ }^{3}$.

Sorensens Definition zeigt die Parallelen zum Thema Wachstumsstrategien. Dieses Thema wiederum ist in der Wissenschaft ausführlicher erforscht als das Thema Business Development ${ }^{4}$. Voeth et al. haben herausgefunden, dass $\mathrm{zu}$ dem Thema Business Development bisher nur sehr wenige Studien veröffentlicht wurden ${ }^{5}$. Außerdem stellen sie fest:

\footnotetext{
${ }^{1}$ Vgl. Kohne 2016, S. 5 und Becker 2014, S. 31.

${ }^{2}$ Vgl. Bernecker 2015, S. 12-14.

${ }^{3} \mathrm{Vgl}$. Sorensen 2012, S. 26.

${ }^{4}$ Vgl. Voeth et al. 2014, S. 6.

${ }^{5}$ Vgl. Voeth et al. 2014, S. 6. 
Tab. 2.1 Übersicht über unterschiedliche Business Development Definitionen

\begin{tabular}{l|l}
\hline Autor(en) (Jahr) & Definition \\
\hline Kohne (2016) & $\begin{array}{l}\text { „Business Development soll: 1. Bestehende, aber unerfüllte } \\
\text { Marktbedürfnisse befriedigen, 2. neue Technologie, Produkte oder } \\
\text { Dienstleistungen auf den Markt bringen, 3. bestehenden Markt mit } \\
\text { neuem Geschäftsmodell verbessern, aufbrechen oder verändern und } \\
\text { 4. ganz neuen Markt schaffen.“a }\end{array}$ \\
\hline Bernecker (2015) & $\begin{array}{l}\text { „Business Development ist die strukturierte und systematische } \\
\text { Weiterentwicklung eines bestehenden oder neuen Geschäftsfeldes. } \\
\text { Als Hauptziele lassen sich Neukundengewinnung, Erschließung } \\
\text { neuer Märkte und Entwicklung neuer Geschäftsmodelle } \\
\text { identifizieren. Die drei Hauptdimensionen des Business } \\
\text { Development sind: Vertrieb-, Produkt-, und Marketingorientiertes } \\
\text { Business Development. “b }\end{array}$ \\
\hline Becker (2014) & $\begin{array}{l}\text { „Die Rolle des Business Development Manager zeichnet sich nach } \\
\text { unserer Definition durch eine oder mehrere der folgenden Aufgaben } \\
\text { aus: } \\
\text { - Konzeption und Implementierung von Strategien in sich } \\
\text { wandelnden Märkten; } \\
\text { - Ausweitung bestehender Marktzugänge und Kanäle (Broadening / } \\
\text { Deepening); } \\
\text { - Erschließen neuer Geschäftsoptionen und -modelle (Business } \\
\text { Innovation).“c }\end{array}$ \\
\hline Sorensen (2012) & $\begin{array}{l}\text { „Business development’ refers to the tasks and processes } \\
\text { concerning analytical preparation of potential growth opportunities, } \\
\text { the support and monitoring of the implementation of growth } \\
\text { opportunities, but does not include decisions on strategy and } \\
\text { implementation of growth opportunities.“d }\end{array}$ \\
\hline
\end{tabular}

Quelle: Eigene Darstellung.

${ }^{a}$ Vgl. Kohne 2016, S. 5.

bVgl. Bernecker 2015, S. 12-14.

${ }^{c}$ Vgl. Becker 2014, S. 31.

dVgl. Sorensen 2012, S. 26.

"Anders als in etablierten Forschungsbereichen der Betriebswirtschaftslehre, in denen vor allem explikative und normative Forschung erforderlich ist, steht die Wissenschaft (und auch die Praxis) beim Business Development noch ganz am Anfang. Hier ist zunächst deskriptive Forschung zu leisten [...]."

\footnotetext{
${ }^{6}$ Voeth et al. 2014, S. 7.
} 
Da Wachstumsstrategien nicht explizit das Thema dieser Dissertation sind, werden im Folgenden nur kurz die vier bekanntesten Wachstumsstrategien von Ansoff vorgestellt (siehe Abb. 2.1). Laut Ansoffs Produkt-Markt-Matrix können Unternehmen Wachstum generieren, indem sie in bestehenden Märkten mit bestehenden Produkten den Markt durchdringen (=Marktdurchdringungsstrategie) ${ }^{7}$. Weiterhin kann ein Unternehmen wachsen, indem es neue Märkte erschließt (=Marktentwicklung) oder neue Produkte entwickelt (=Produktentwicklung). Schlussendlich besteht für ein Unternehmen auch die Möglichkeit neue Märkte mit neuen Produkten zu erschließen (=Diversifikation). ${ }^{8}$

\begin{tabular}{|c|c|c|c|c|c|}
\hline Produkte & MO & M1 & M2 & $\ldots$ & $\mathrm{Mm}$ \\
\hline PO & $\begin{array}{l}\text { Marktdurch- } \\
\text { dringung }\end{array}$ & \multicolumn{2}{|c|}{ Marktentwicklung } & & \\
\hline P1 & \multirow{3}{*}{ 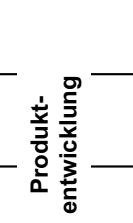 } & & & & \\
\hline P2 & & \multicolumn{2}{|c|}{ Diversifikation } & & \\
\hline$\vdots$ & & & & & \\
\hline$P n$ & & & & & \\
\hline
\end{tabular}

Abb. 2.1 Produkt-Markt-Matrix. (Quelle: Eigene Darstellung in Anlehnung an Ansoff 1957, S. 114)

Weiterhin kann Wachstum sowohl unternehmensintern als auch unternehmensextern stattfinden. Unternehmensinternes Wachstum wird durch die externe oder interne Finanzierung von Geschäften generiert. Unternehmensexternes Wachstum findet statt, durch Fusionen oder durch die Übernahme von Unternehmen. ${ }^{9}$ Um erfolgreich zu wachsen, müssen zahlreiche Faktoren beachtet werden, die hier nicht im Fokus stehen. Eine Internationalisierungsstrategie beschäftigt sich demnach mit der Marktentwicklung oder der Diversifikation.

\footnotetext{
${ }^{7}$ Vgl. Ansoff 1957, S. 114.

${ }^{8}$ Vgl. Ansoff 1957, S. 114.

${ }^{9} \mathrm{Vgl}$. Weston 1953, S. 3.
} 
Die Unterschiede zwischen Business Development und Wachstumsstrategien sind, dass die neuen Modelle, d. h. Business Development Modelle, konkretere Ansätze geben wie neues Wachstum geschaffen werden kann. Zum Beispiel gibt es das Business Model Canvas ${ }^{10}$ oder den St. Galler Business Model Navigator ${ }^{11}$. Diese beiden Modelle tragen zur Geschäftsfeldentwicklung bei. Das ISA-Modell kann der Dimension "neue Märkte" von Bernecker zugeordnet werden, wohingegen die anderen beiden Modelle der Neukundengewinnungsdimension und der Geschäftsmodelldimension zugeordnet werden können. Somit reiht sich das ISA-Modell als weiteres Modell in die Business Development-Modelle ein.

Das Thema der vorliegenden Dissertation kann in den Internationalisierungstheorien weiter verortet werden. Holtbrügge \& Welge z. B. unterscheiden dabei zwischen drei theorieübergreifenden Ansätzen: Außenhandelstheorien, Theorien der internationalen Direktinvestition und Theorien der Multinationalen Unternehmung ${ }^{12}$. Während die Außenhandelstheorien die Vorteilhaftigkeit des Außenhandels an sich untersuchen, beschäftigen sich die Theorien der internationalen Direktinvestitionen mit den Voraussetzungen für eine Internationalisierung der Geschäfte. Die neueren Theorien der multinationalen Unternehmung untersuchen dagegen, warum manche Unternehmen Wettbewerbsvorteile aus einer Internationalisierung gewinnen können und andere Unternehmen nicht. ${ }^{13}$ Insgesamt lässt sich festhalten, dass fast alle Ansätze sich mehr oder weniger mit der Frage beschäftigen, warum Unternehmen ausländische Märkte erschließen, nicht aber wie Unternehmen bei ihrem Internationalisierungsprozess vorgehen ${ }^{14}$. Johanson \& Vahlne's Lerntheorie der Internationalisierung ist eine Ausnahme. Die Autoren beschreiben z. B., dass Unternehmen in der Regel einen neuen Ländermarkt mit Exporten über einen Handelspartner erschließen. Wenn die Geschäfte gut laufen, folgen Vertriebsniederlassungen bzw. Produktionsniederlassungen. ${ }^{15}$

Laut einer Zusammenstellung vom Lehrstuhl für Vertriebsmanagement und Business-to-Business Marketing der WHU gibt es ca. 30 Lehrstühle an deutschen Universitäten und Fachhochschulen, die sich mit dem Thema Verkaufsund Vertriebsmanagement beschäftigen ${ }^{16}$. In der Regel sind dies Lehrstühle für Marketing. Eine Ausnahme bildet der Lehrstuhl von Prof. Dr. Ivens, Lehrstuhl

\footnotetext{
${ }^{10}$ Vgl. Osterwalder \& Pigneur 2011, S. 48 ff.

${ }^{11} \mathrm{Vgl}$. Gassmann et al. 2014, S. $81 \mathrm{ff}$.

${ }^{12}$ Holtbrügge \& Welge 2015, S. $55 \mathrm{ff}$.

${ }^{13}$ Vgl. Holtbrügge \& Welge 2015, S. 55.

${ }^{14}$ Vgl. Holtbrügge \& Welge 2015, S. 83.

${ }^{15}$ Vgl. Johanson \& Vahlne 1977.

${ }^{16}$ Vgl. Lehrstuhl für Vertriebsmanagement und Business-to-Business Marketing 2013.
} 
für BWL, insb. Vertrieb und Marketing an der Universität Bamberg und Prof. Dr. Jensen, Lehrstuhl für Vertriebsmanagement und Business-to-Business Marketing. ${ }^{17}$ Binckebanck \& Hölters kommen daher in einer Aufarbeitung zum Stand der Forschung zum internationalen Vertrieb zu dem Ergebnis, dass das Thema in der Vergangenheit weniger Beachtung erhalten hat ${ }^{18}$. Mögliche Gründe sind, zum einen, dass der Vertrieb in der Regel sehr praxisorientiert ist, d. h. um das Vertriebsgeschäft zu verstehen, müssen industriebezogene Marktkenntnisse vorliegen. Zum anderen wurde der Vertrieb auch lange Zeit anderen Wissenschaftsbereichen, wie z. B. dem strategischen Management, dem Logistikmanagement oder dem Betriebsführungsmanagement überlassen ${ }^{19}$. So gibt es z. B. im strategischen Marketing die Konzepte der generischen Wertschöpfungskette ${ }^{20}$, Wertsystem ${ }^{21}$, herstellerbezogene und käuferbezogene Produktketten ${ }^{22}$, globale Wertschöpfungskette $^{23}$ und Wertschöpfungslächeln ${ }^{24}$ die allesamt dem Vertrieb bzw. seinen Akteuren eine strategische Rolle zuordnen. Insbesondere das Wertsystem (siehe Abb. 2.2) von Porter zeigt, dass Wettbewerbsvorteile nur im Zusammenhang mit dem Verständnis dieses Systems für ein bestimmtes Unternehmen generiert werden können ${ }^{25}$.

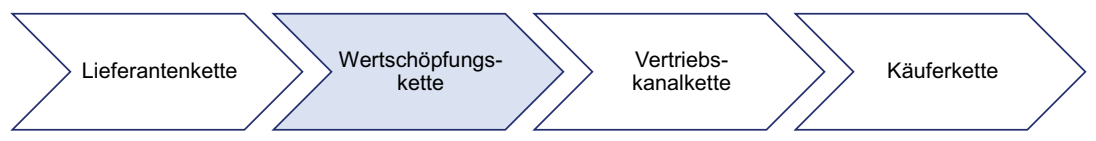

Abb. 2.2 Das Wertsystem. (Quelle: Eigene Darstellung in Anlehnung an Porter 1985, S. 35)

Weitere Konzepte stammen aus dem Logistikmanagement (siehe Abb. 2.3) und dem Betriebsführungsmanagement (siehe Abb. 2.4). In Abb. 2.3 ist der Prozess

${ }^{17}$ Vgl. Lehrstuhl für Vertriebsmanagement und Business-to-Business Marketing 2013, S. 5-8.

${ }^{18}$ Vgl. Binckebanck \& Hölter 2012, S. 223-240.

${ }^{19}$ Vgl. z. B. Balambo et al. 2014, S. 74.

${ }^{20}$ Vgl. Porter 1985, S. 37 und Porter 1986, S. 18.

${ }^{21} \mathrm{Vgl}$. Porter 1985, S. 35.

${ }^{22} \mathrm{Vgl}$. Gereffi 1994, S. 97.

${ }^{23} \mathrm{Vgl}$. Gereffi et al. 2005, S. 89.

${ }^{24}$ Vgl. Bartlett \& Ghoshal 2000, Mudambi 2007, S. 206 und Lundquist 2007, S. 56.

${ }^{25}$ Vgl. Porter 1985, S. 34. 
des Logistikmanagements abgebildet. Die Grenzen der Logistik werden anhand der gepunkteten Linie dargestellt und liegen innerhalb eines Unternehmens.

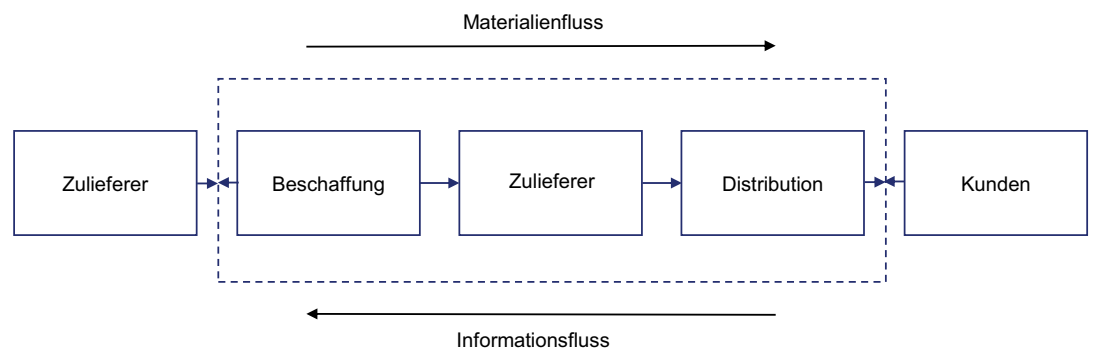

Abb. 2.3 Der Prozess des Logistikmanagements. (Quelle: Eigene Darstellung in Anlehnung an Christopher 2011, S. 11)

Das Logistikmanagement stellt sicher, dass die Materialien oder Produkte transportiert werden und dass Informationen an die entsprechenden Abteilungen, wie z. B. Marketing berichtet werden. ${ }^{26}$ Ohne geeignete Infrastruktur fällt es Unternehmen unter Umständen schwer Produkte an die Kunden zu bringen ${ }^{27}$.

Abb. 2.4 zeigt den Prozess des Lieferkettenmanagements. Es gibt dabei vorgeschaltete "upstream" Beziehungen zu Rohstofflieferanten und Komponentenzulieferern sowie nachgeschaltete "downstream" Beziehungen zu Großhändlern, Einzelhändlern und Kunden ${ }^{28}$.

Insgesamt zeigt sich, dass das Thema internationaler Vertrieb sehr interdisziplinär und durch unterschiedliche Forschungseinflüsse geprägt ist. Aus dieser Interdisziplinarität lässt sich erklären, warum der internationale Vertrieb in der Marketingliteratur in der Vergangenheit weniger Beachtung bekommen hat als z. B. die anderen drei Marketing-Mix Elemente ${ }^{29}$.

In den letzten Jahren wurden deshalb in der Marketingliteratur vermehrt wissenschaftliche Artikel mit dem Aufruf zur Schließung von Forschungslücken im internationalen Verkaufs- und Vertriebsmanagement veröffentlicht. Tab. 2.2 gibt

\footnotetext{
${ }^{26}$ Vgl. Christopher 2011, S. 11.

${ }^{27}$ Vgl. Interview 442017 und Interview 452017.

${ }^{28}$ Vgl. Christopher 2011, S. 3.

${ }^{29}$ Vgl. z. B. Homburg \& Krohmers 2006 Seitenverhältnis der Marketing-Mix Elemente. Die Vertriebspolitik umfasst im Vergleich zu den anderen drei Marketing-Mix Elementen ca. die Hälfte der Seitenzahlen.
} 


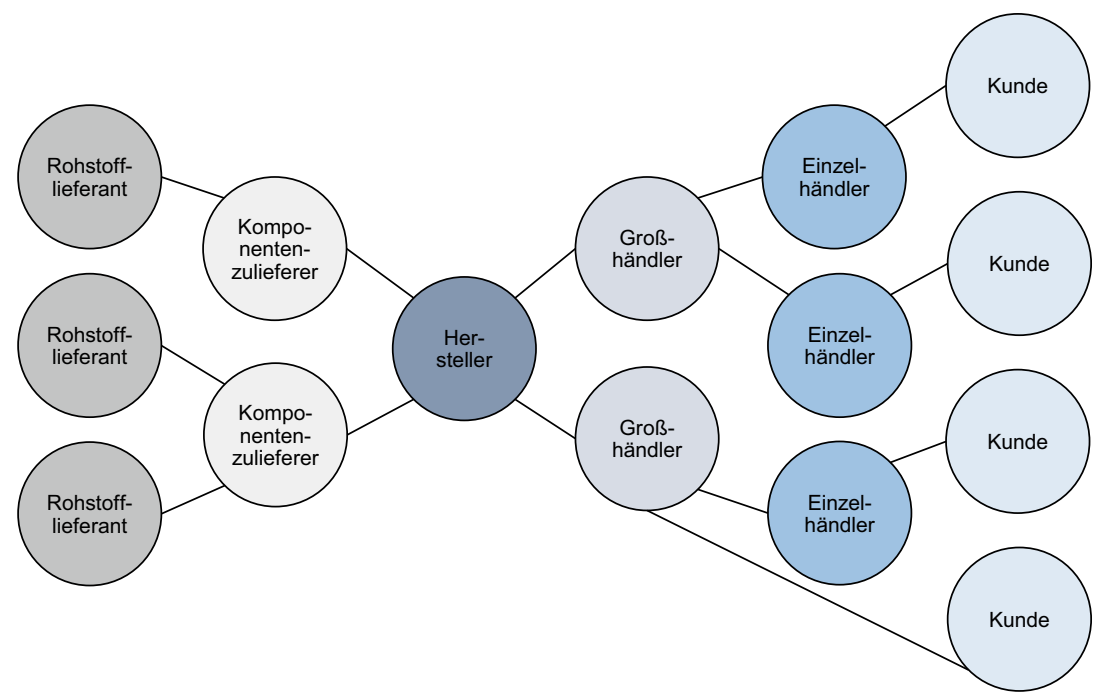

Abb. 2.4 Der Prozess des Lieferkettenmanagements. (Quelle: Eigene Darstellung in Anlehnung an Kummer et al. 2009. S. 260)

eine Übersicht über die aktuellen Forschungslücken. Baldauf \& Lee rufen z. B. zu mehr Forschung außerhalb der USA auf sowie zur Entwicklung von neuen Modellen und Theorien ${ }^{30}$. Panagopoulos et al. 's Aufsatz ist ein Gemeinschaftswerk von insgesamt 13 Wissenschaftlern ${ }^{31}$. In dem Aufsatz erfolgt eine dementsprechende Auswertung der bisherigen Forschungsaufsätze in dem Themengebiet. Sie stellen einige Forschungslücken vor, z. B. sollten sich zukünftige Forschungsprojekte mit dem Vertrieb in Schwellenländern und Entwicklungsländern beschäftigen. Außerdem mangelt es an neuen theoretischen Modellen für Praktiker oder Erkenntnissen über das Schnittstellenmanagement zwischen Vertrieb und anderen funktionellen Gebieten. ${ }^{32}$ Wagner \& Szymura-Tyl erarbeiten ebenfalls eine Forschungslücke in Bezug auf das Schnittstellenmanagement zwischen Vertrieb, Marketing und Neuproduktentwicklung, sowie einer Aufforderung zur Untersuchung der Auswirkung einer Verbesserung der Vertriebsorganisation auf das strategische Management. ${ }^{33}$

\footnotetext{
${ }^{30}$ Vgl. Baldauf \& Lee 2011, S. 212 ff.

${ }^{31} \mathrm{Vgl}$. Panagopoulos et al. 2011.

${ }^{32}$ Vgl. Panagopoulos et al. 2011, S. 226 f.

${ }^{33}$ Vgl. Wagner \& Szymura-Tyl 2016, S. 3619 f.
} 
Tab. 2.2 Übersicht Forschungslücken Internationales Verkaufs- und Vertriebsmanagement

\begin{tabular}{l|l}
\hline Autor(en) und Jahr & Forschungslücken \\
\hline Baldauf \& Lee 2011 & - Durchführung von Forschung außerhalb der USA \\
& - Entwicklung von neuen Modellen und Theorien \\
\hline Panagopoulos et al. 2011 & - Durchführung von Forschung, die sich auf den \\
& Vertrieb in Schwellenländern und weniger \\
& entwickelten Ländern konzentriert \\
& - Identifizierung und Verifizierung von \\
& Vertriebsmanagementvariablen \\
& - Entwicklung von neuen theoretischen Gerüsten \\
& - Identifizierung von Wissens- und \\
& Informationsstrukturen aus ausländischen Märkten und \\
& deren Aufbaumöglichkeiten \\
& - Betrachtung der Schnittstellen zwischen dem Vertrieb \\
& und anderen funktionellen Gebieten \\
& - Verbesserung des Verständnisses von \\
& Exportprozeduren und Bürokratie im Ausland \\
\hline Wagner \& Szymura-Tyl 2016 & Betrachtung von Schnittstellen zwischen Vertrieb, \\
& Marketing und Neuproduktentwicklung \\
& - Verbesserung der Vertriebsorganisation innerhalb eines \\
& Unternehmens und deren Einfluss auf das Strategische \\
& Management \\
\hline
\end{tabular}

Quelle: Eigene Darstellung.

Weitere Forscher bekräftigen z. B., dass der Vertrieb eine Quelle für Marktdaten ist und diese Quelle im Unternehmen zur Sammlung von Wettbewerbsinformationen und rechtlichen Regulierungen genutzt werden soll ${ }^{34}$. Samli et al. heben ebenfalls die strategische Bedeutung des Vertriebs hervor ${ }^{35}$. Leach et al. beschäftigen sich ausschließlich mit dem Thema der Distribution ${ }^{36}$, wohingegen Lancioni oder LaRoche et al. den Vertrieb in Verbindung zur Preispolitik und der Kommunikationspolitik bringen ${ }^{37}$. Leach et al. argumentieren, dass die Macht welche die Hersteller oder Einzelhändler über Vertriebskanäle haben, einen starken Einfluss auf die Wahl eines Vertriebskanals hat ${ }^{38}$. Einer der wenigen neueren

\footnotetext{
${ }^{34}$ Vgl. Chonko et al. 1991.

${ }^{35} \mathrm{Vgl}$. Samli et al. 1994.

${ }^{36} \mathrm{Vgl}$. Leach et al. 2011.

${ }^{37}$ Vgl. Lancioni 2005 und LaRoche et al. 2005.

${ }^{38}$ Vgl. Leach et al. 2011, S. 217.
} 
Artikel beschäftigt sich mit dem Vertriebsmanagement in Südkorea ${ }^{39}$. Weitere wissenschaftliche Artikel lassen sich unter folgenden Themen zusammenfassen: Organisation bzw. Management des Vertriebs ${ }^{40}$, Vertriebscontrolling ${ }^{41}$, Personalmanagement im Vertrieb ${ }^{42}$, Einfluss der Kultur auf den Vertrieb ${ }^{43}$ oder Customer Relationship Management ${ }^{44}$. Die vorliegende Arbeit trägt dazu bei die aufgezeigten Forschungslücken zu füllen. Außerdem lässt sich die Arbeit dem strategischen Vertrieb zuordnen.

\subsection{Zur Entwicklung der Methodik des International Sales Accelerator Modells}

Das ISA-Modell wurde aufgrund der aufgezeigten Forschungslücken (siehe Abschnitt 2.1) und eigenen Beobachtungen von unsystematischen Vorgehensweisen von Internationalisierungsprozessen bei Unternehmen entwickelt. Es ist ein ganzheitlicher Ansatz mit insgesamt sieben Baukästen zur Entwicklung und Implementierung von Internationalisierungsentscheidungen (siehe Abb. 2.5) ${ }^{45}$. Dabei ist das ISA-Modell theoretisch fundiert und beinhaltet zahlreiche Erkenntnisse aus der wissenschaftlichen Literatur, auf die in den nachfolgenden Abschnitten näher eingegangen wird.

Dem ISA-Modell liegt der Prozess des strategischen Managements mit den drei Phasen - strategische Analyse, Strategieformulierung und Strategieauswahl und Strategieimplementierung - zugrunde (siehe Abb. 2.6) ${ }^{46}$.

Die Phase der strategischen Analyse beinhaltet die ersten drei Schritte des ISA-Modells. In Schritt vier findet die Strategieformulierung und Strategieauswahl statt. Anschließend erfolgt in den Schritten fünf bis sieben des ISA-Modells die Strategieimplementierung. Weiterhin versteht sich das ISA-Modell als ein

\footnotetext{
${ }^{39}$ Vgl. Horak \& Nihalani 2016.

${ }^{40}$ Vgl. Gestetner 1974, Baldauf et al. 2001a, Baldauf et al. 2001b oder Baldauf et al. 2002.

${ }^{41}$ Vgl. Jacobs \& Larkins 1992, Hill \& Allaway 1993, Black et al. 1998, Peasnell 1998, Cravens et al. 2006 oder Borgonovo \& Peccati 2007.

${ }^{42}$ Vgl. Honeycutt \& Ford 1996, Wetzels et al. 1999 oder Mallin et al. 2010.

${ }^{43}$ Vgl. DeShields \& de los Santos 2000.

${ }^{44}$ Vgl. Davies et al. 2010 oder Baker 2014.

${ }^{45}$ Der ganzheitliche Ansatz des ISA-Modells knüpft an Kochs Erkenntnis an, dass die internationale Marktauswahl und Markteintrittsstrategien kein separater Prozess sind, sondern zwei Aspekte einer Entscheidung (Vgl. Koch 2001, S. 73).

${ }^{46}$ Vgl. Hungenberg 2014, S. 9.
} 
Strategischer Markteintritt

Operative Schritte der

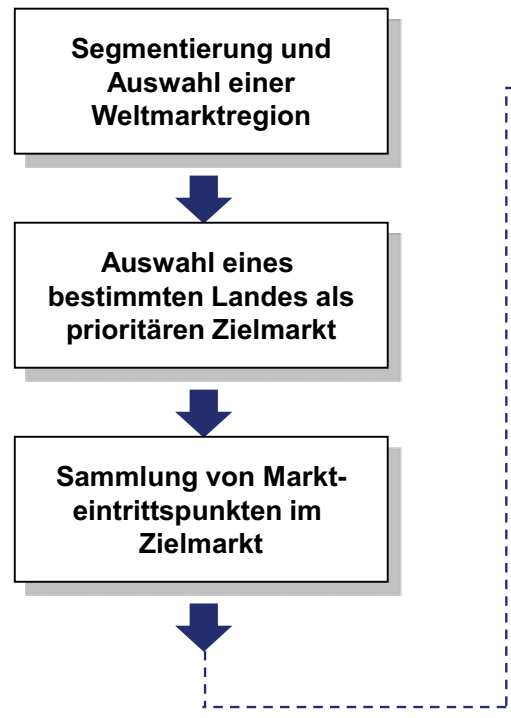

Markterschließung

Entwicklung und

Entscheidung

für eine Markteintritts-

strategie

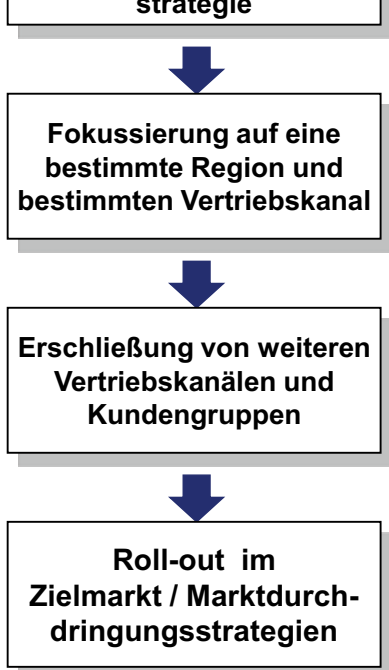

Abb. 2.5 Das International Sales Accelerator-Modell. (Quelle: Eigene Darstellung)

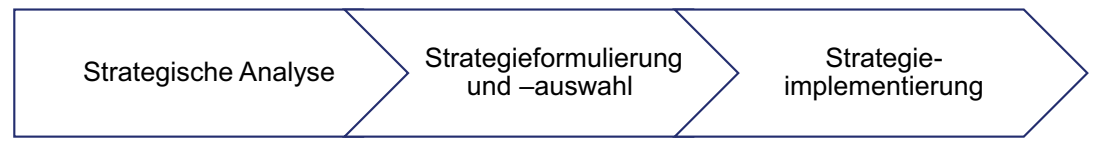

Abb. 2.6 Prozess des strategischen Managements. (Quelle: Eigene Darstellung)

erklärendes Modell $^{47}$, das eine Abbildung eines Realitätsausschnitts von Best Practices darstellt und Unternehmen und Institutionen bei der Planung und Implementierung von Internationalisierungsprozessen dient.

${ }^{47}$ Manhart 1995, S. 14 f. 


\section{Baustein 1: Segmentierung und Auswahl einer Weltregion}

Im ersten Baustein gilt es Antworten auf bestimmte Kernfragen zu Schritt 1 (siehe Abb. 2.7) abzuklären. Eine erste Kernfrage lautet: "Aus welchen Gründen wollen wir international tätig werden?" Welge \& Holtbrügge listen die folgenden vier Motive auf: markt- und absatzorientierte Motive, kosten- und ertragsorientierte Motive, beschaffungsorientierte Motive und strategische Motive ${ }^{48}$. Marktund absatzorientierte Motive sind z. B. gegeben, wenn ein Unternehmen eine Sättigung im Produktlebenszyklus eines Produktes im Heimatmarkt wahrnimmt und diese durch eine Internationalisierung der Geschäfte zu umgehen versucht. Manche Staaten fördern auch die Ansiedlung von ausländischen Unternehmen. In diesem Fall liegt ein kosten- und ertragsorientiertes Motiv vor. Weiterhin treten Unternehmen in neue Ländermärkte ein, weil sie z. B. Ressourcen vor Ort sicherstellen wollen. Das wäre ein beschaffungsorientiertes Motiv. Zu guter Letzt können Unternehmen mit einer Internationalisierungsstrategie auch strategische Ziele verfolgen, wie z. B. der Aufkauf eines Konkurrenten im Ausland. ${ }^{49}$

\begin{tabular}{|c|c|}
\hline & $\begin{array}{c}- \text { Aus welchen Gründen wollen wir international tätig werden? } \\
- \text { Haben wir die nötigen Ressourcen und Kompetenzen, um } \\
\text { Geschäfte außerhalb unserer Region aufzubauen? }\end{array}$ \\
\hdashline & - Wollen wir Geschäfte außerhalb unserer Weltregion tätigen? \\
\hdashline & Welche Weltregionen werden in unserer Industrie am schnellsten \\
\hdashline
\end{tabular}

Abb. 2.7 Kernfragen zu Schritt 1. (Quelle: Eigene Darstellung)

Eine weitere Kernfrage in Schritt 1 lautet: "Haben wir die nötigen Ressourcen und Kompetenzen, um Geschäfte außerhalb unserer Region aufzubauen?" Akhter \& Robles betonen, dass Manager zunächst analysieren sollten, welche Kompetenzen sich auf andere Ländermärkte übertragen lassen, bevor sie überhaupt eine Internationalisierung in Betracht ziehen ${ }^{50}$. Weiterhin müssen Manager sich die Frage stellen: "Wollen wir Geschäfte außerhalb unserer Weltregion tätigen?" Die Kernfrage rührt daher, dass laut Johansson \& Vahlne's Lerntheorie der Internationalisierung Unternehmen in der Regel zunächst in ihrer Weltregion

\footnotetext{
${ }^{48}$ Vgl. Welge \& Holtbrügge 2006, S. 24.

${ }^{49}$ Vgl. Welge \& Holtbrügge 2006, S. 24.

${ }^{50}$ Vgl. Akther \& Robles 2006.
} 
international tätig werden, bevor sie in andere Weltregionen expandieren ${ }^{51} . \mathrm{Zu}$ guter Letzt sollten sich Manager die Frage stellen: "Welche Weltregionen werden in unserer Industrie am schnellsten wachsen?" Hierzu können Manager z. B. Handelsdaten heranziehen, um die wachstumsstärksten Weltregionen für ihre Industrie herauszufinden (siehe auch Fallstudie 6.1).

\section{Baustein 2: Auswahl eines bestimmten Landes als prioritären Zielmarkt}

Nach einer ersten Segmentierung und Auswahl einer Weltregion geht es im zweiten Baustein des ISA-Modells um die Auswahl eines bestimmten Landes als prioritären Zielmarkt. Bei ersten Vorabinterviews fiel dabei auf, dass die Entscheidung für einen primären Zielmarkt in der Praxis oft unsystematisch und je nach Ländermarkt unterschiedlich getätigt wurde. Dabei argumentieren mehrere Forscher für ein systematisches Vorgehen bei der internationalen Marktauswahl ${ }^{52}$. Brouthers \& Nakos liefern zudem erste empirische Beweise, dass ein systematisches Vorgehen bei der internationalen Marktauswahl in höheren Exportleistungen von KMUs mündet ${ }^{53}$. Außerdem sollten sich Manager Gedanken zu den in Abb. 2.8 aufgelisteten Kernfragen zu Schritt 2 machen.

\footnotetext{
- Mit welcher Methode sollen die Länder ausgewählt werden (Marktschätzungs- oder Marktgruppierungsverfahren)?

- Welche Länder innerhalb einer Weltregion sollen zuerst erschlossen werden?

- In welchem Land soll das Headquarter für die Region aufgebaut werden?
}

Abb. 2.8 Kernfragen zu Schritt 2. (Quelle: Eigene Darstellung)

Eine erste Kernfrage in Schritt 2 lautet: "Mit welcher Methode (Marktschätzungs- und Marktgruppierungsverfahren) sollen die Länder ausgewählt werden?" Die Unterscheidung der zwei grundlegenden Verfahren stammt von Papadopoulos \& Denis ${ }^{54}$. Der Unterschied zwischen den zwei Vorgehensweisen besteht darin, dass Länder beim Marktgruppierungsverfahren anhand von

\footnotetext{
${ }^{51}$ Vgl. Johansson \& Vahlne 1977.

${ }^{52}$ Vgl. z. B. Kobrin 1979 und Andersen \& Buvik 2002.

${ }^{53}$ Vgl. Brouthers \& Nakos 2005, S. 376.

${ }^{54}$ Vgl. Papadopoulos \& Denis 1988, p. 40.
} 
Clustern ausgewählt werden wohingegen Länder beim Marktschätzungsverfahren vor der Auswahl in eine bestimmte Reihenfolge gebracht werden ${ }^{55}$. Eine Einteilung weiterer seit 1988 veröffentlichten Studien zum Thema internationale Marktauswahl kann Tab. 5.3 in Abschnitt 5.1. dieser Arbeit entnommen werden. Generell kann bei der Literaturanalyse beobachtet werden, dass die Faktoren für die internationale Marktauswahl stetig gestiegen sind. Praktiker stellt dies vor eine Herausforderung, da sie sich erstmal eine Übersicht über die relevanten Faktoren verschaffen müssen und dies in der Regel mit Zeit und Ressourcen verbunden ist. In Tab. 2.3 sind einige ausgewählte Studien und deren Faktorenauswahl für die internationale Marktauswahl dargestellt. Zudem kann der interessierte Leser in Abschnitt 6.1 mehr über eine internationale Marktauswahl mit Hilfe von Handelsdaten erfahren. Das Verfahren in Abschnitt 6.1.1 wurde von der Autorin der vorliegenden Dissertation weiterentwickelt und kann den Marktschätzungsverfahren zugeteilt werden ${ }^{56}$.

Johansson \& Vahlne argumentieren dafür, dass Unternehmen neue Märkte basierend auf dem industriellen Netzwerk eines Unternehmens auswählen ${ }^{57}$. Andere Forscher betonen die Bedeutung der Wettbewerbsposition eines Unternehmens und der Marktattraktivität eines bestimmten Produkts ${ }^{58}$. In neueren Studien wird versucht mehrere Faktoren einzubeziehen oder verschiedene Theorien in ihre Studien aufzunehmen. Zum Beispiel, Brouthers et al. operationalisieren Dunnings OLI-Paradigma zur Auswahl neuer Märkte ${ }^{59}$. Martín \& Drogendijk hingegen bauen zahlreiche Distanzmaße in ihre Studie ein, um neue Märkte auszuwählen ${ }^{60}$.

Eine weitere Kernfrage in Schritt 2 lautet: "Welche Länder innerhalb einer Weltregion sollen zuerst erschlossen werden?" Die Frage stellt sich insbesondere bei den Marktgruppierungsverfahren und bei Verfahren, die keine Gruppierung und kein Ranking erstellen (siehe Tab. 5.3 in Abschnitt 5.1). Hier bietet es sich an eine Roadmap für die Erschließung einer Weltregion zu entwickeln, in dem die nachstehenden ISA-Modell Schritte für die jeweiligen Ländermärkte aufgezeigt werden und kontinuierlich ergänzt werden. Zu guter Letzt stellt sich die Frage "In welchem Land soll das Headquarter für die Region aufgebaut werden?". Viele der

\footnotetext{
${ }^{55}$ Vgl. Papadopoulos \& Denis 1988, p. 39-44.

${ }^{56}$ Das Marktauswahlverfahren "Export-GAP Analyse" in Kapitel 6.1 basiert auf einem Auswahlverfahren (Export.gov 2013) der Agentur International Trade Administration des US-Handelsministeriums, welches durch die Autorin weiterentwickelt wurde.

${ }^{57}$ Vgl. Johanson \& Vahlne 1990, S. 18.

${ }^{58}$ Vgl. Attiyeh \& Werner 1981, S. 79f und Brewer 2001, S. 169.

${ }^{59}$ Vgl. Brouthers et al. 2009, S. 272.

${ }^{60}$ Vgl. Martín \& Drogendijk 2014, S. 107.
} 


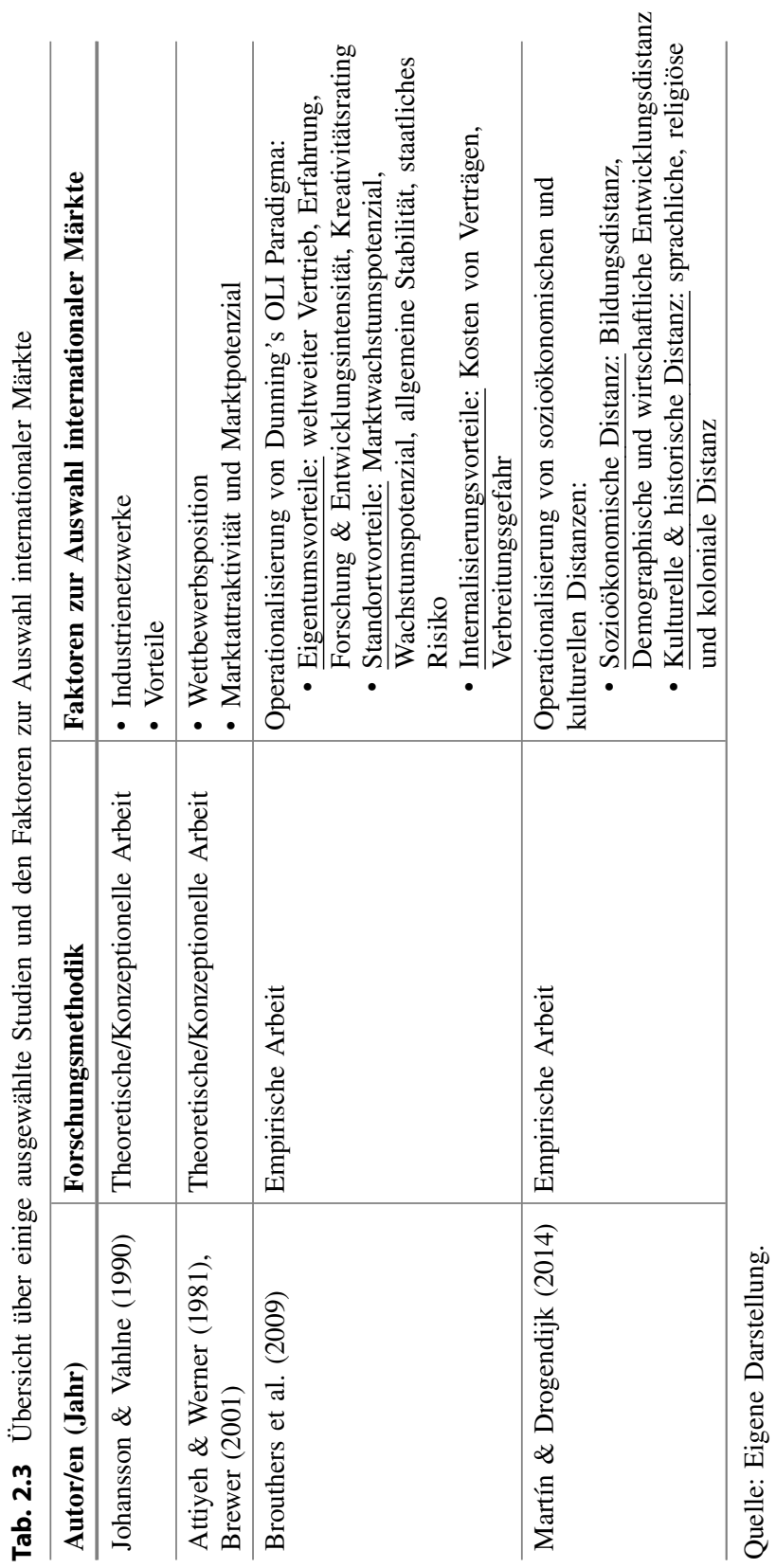


befragten Unternehmen bestätigten, dass ein Headquarter für eine Region festgelegt wurde ${ }^{61}$. Die Auswahl eines Standortes für diese Headquarter sollte auch die Betrachtung politischer und kultureller Gesichtspunkte beinhalten ${ }^{62}$.

\section{Baustein 3: Sammlung von Markteintrittspunkten im Zielmarkt}

Im dritten Baustein des ISA-Modells müssen Module zur ersten Kontaktaufnahme mit einem Ländermarkt ausgewählt werden. In der Literatur werden z. B. in Handelszeitschriften die Handelskammern als Markteintrittspunkte beschrieben ${ }^{63}$. Anthony bezeichnet als Markteintrittspunkt die Entscheidung welche Kunden angesprochen werden, z. B. der untere oder der mittlere Teil der Kundenpyramide in Schwellenländern ${ }^{64}$. Eine relativ große Anzahl an Autoren versteht darunter den Markteintrittszeitpunkt, d. h. zu welchem Zeitpunkt ein Markt erschlossen wird $^{65}$. Dazu gibt es zwei Strategien mit zwei unterschiedlichen Betrachtungsweisen: Die länderübergreifende sowie die länderspezifische Wasserfall- und Sprinklerstrategie. Allerdings verstehen die Autoren unter den beiden Strategien nicht den Markteintrittszeitpunkt in ein Land, sondern vielmehr die Reihenfolge der Bearbeitung der ausgewählten Zielmärkte (länderübergreifend) sowie die Reihenfolge der Bearbeitung eines Marktes im Vergleich zu den Wettbewerbern (länderspezifisch). ${ }^{66}$ Sandberg beschäftigt sich mit sogenannten Markteintrittsknoten: Markteintrittsknoten sind Einrichtungspunkte in ein Netzwerk ausländischer Märkte ${ }^{67}$. Bei näherer Betrachtung haben für Sandberg Markteintrittsknoten letztendlich eine ähnliche Bedeutung wie Markteintrittsstrategien (siehe Kapitel 4$)^{68}$. Daher lässt sich festhalten, dass Schritt 3 des ISA-Modells in der Literatur bisher ebenfalls, wie Schritt 1 des ISA-Modells, kaum beschrieben wird. In Abb. 2.9 sind die wichtigsten Kernfragen zu Schritt 3 zusammengefasst.

Zunächst sollten sich Manager fragen: "Welche Geschäftspartner kennen wir bereits im Zielmarkt?" Eventuell gibt es befreundete Unternehmen, die erste wichtige Informationen zum Zielmarkt zur Verfügung stellen können. Weiterhin gilt es herauszufinden: "Wer sind die wichtigsten Promotoren für dieses Land im eigenen

\footnotetext{
${ }^{61}$ Vgl. z. B. Interview 412017 und Interview 472018.

${ }^{62}$ Vgl. z. B. Interview 472018.

${ }^{63}$ Vgl. Caribbean Business 2004, S. 48.

${ }^{64} \mathrm{Vgl}$. Anthony 2012.

${ }^{65}$ Vgl. z. B. Backhaus et al. 2000, S. 127 oder Meffert \& Pues 2002, S. 403 ff.

${ }^{66}$ Vgl. Meffert \& Pues 2002, S. 403 ff.

${ }^{67}$ Vgl. Sandberg 2013, S. 106.

${ }^{68}$ Vgl. Sandberg 2013, S. 121.
} 


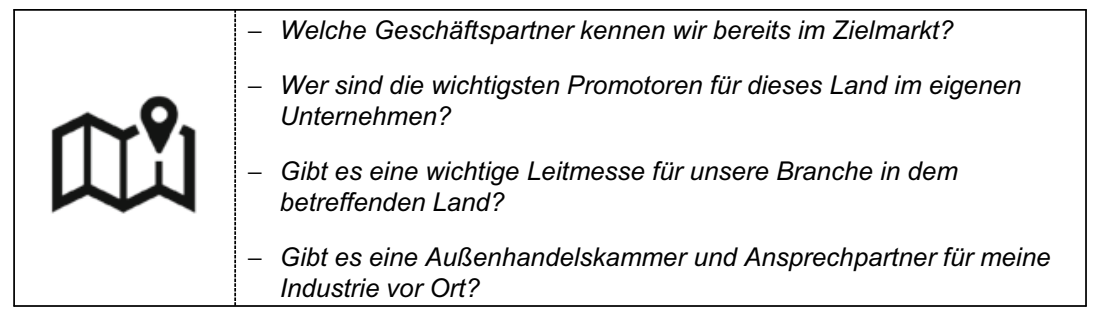

Abb. 2.9 Kernfragen zu Schritt 3. (Quelle: Eigene Darstellung)

Unternehmen?" Ein Interviewpartner aus einem deutschen Umwelttechnikunternehmen berichtete z. B., dass sie den spanischen Markt aufgrund eines wichtigen Promotors im Unternehmen ausgewählt haben, der die Markterschließung im Land vorangetrieben hat ${ }^{69}$. Darüber hinaus gilt es herauszufinden: "Gibt es eine wichtige Leitmesse für unsere Branche in dem betreffenden Land?" und "Gibt es eine Außenhandelskammer und Ansprechpartner für meine Industrie vor Ort?". Wichtige Leitmessen lassen sich über bestimmte Webseiten (siehe Fallstudie im Abschnitt 6.1) herausfinden. Außenhandelskammern können Unternehmen unter anderem im Hinblick auf Schritt 4 des ISA-Modells, den Markteintrittsstrategien, beraten und erste Kontakte zu lokalen Unternehmen herstellen.

\section{Baustein 4: Entwicklung und Entscheidung für eine Markteintrittsstrate-} gie

Im vierten Baustein des ISA-Modells geht es um die Formulierung und Auswahl einer Markteintrittsstrategie. Auch gilt es auf bestimmte Kernfragen eine Antwort zu finden (siehe Abb. 2.10).

Zunächst sollten Manager ihre eigene Unternehmensstrategie analysieren und sich die Frage stellen: "Welche Markteintrittsstrategie passt zu unserer Unternehmensstrategie?" Nach Hill gibt es z. B. vier unterschiedliche internationale Unternehmensstrategien: internationale, globale, multinationale und transnationale Unternehmensstrategie ${ }^{70}$. Die Unternehmensstrategien lassen sich anhand von zwei Dimensionen unterteilen: Grad der Standardisierung sowie Grad der

\footnotetext{
${ }^{69} \mathrm{Vgl}$. Interview 3.

${ }^{70}$ Vgl. Hill 2013, S. 435.
} 
- Welche Markteintrittsstrategie passt zu unserer
Unternehmensstrategie?
- Welche Ziele haben wir für das Zielland für die ersten 2
Jahre?
- Können wir erste Vereinbarungen mit Schlüsselkunden
abschließen?
- Welche Handelsabkommen sind insb. bei
Produktionsniederlassungen zu beachten?

Abb. 2.10 Kernfragen zu Schritt 4. (Quelle: Eigene Darstellung)

Lokalisierung $^{71}$. Belz \& Reinhold argumentieren, dass sich die Markteintrittsstrategien in diese Unternehmensstrategien einordnen lassen ${ }^{72}$. Abb. 2.11 zeigt eine solche Einordnung der Markteintrittsstrategien unter Verwendung von weiteren Markteintrittsstrategien nach Kutschker \& Schmid ${ }^{73}$. Holtbrügge \& Welge weisen darauf hin, dass Unternehmen meistens mit risikoarmen Markteintrittsstrategien beginnen, wie z. B. dem Export. Hill empfiehlt eine Weiterentwicklung der internationalen Unternehmensstrategie in Richtung global, multinational oder transnational $^{74}$. Bei einer multinationalen Unternehmensstrategie empfiehlt sich z. B. eine Markteintrittsstrategie in Kooperation mit einem ausländischen Unternehmen, in Form einer strategischen Allianz oder eines Joint Ventures. Bei diesen Markteintrittsstrategien können die Partner bei der lokalen Anpassung der Produkte von großer Hilfe sein. Verfolgt ein Unternehmen eine globale Unternehmensstrategie stehen z. B. Franchising, Lizenzierungen, Vertragsfertigung oder Managementverträge als Markteintrittsstrategien zur Verfügung. Diese Markteintrittsstrategien helfen Kosten zu sparen und bieten sich insbesondere bei Produkten an, die einen hohen Standardisierungsgrad haben. Die Königsdisziplin im internationalen Geschäft ist die transnationale Unternehmensstrategie. Hier stehen z. B. Tochtergesellschaften, Konsortien, Generalunternehmerschaften oder Betreibermodell zur Auswahl. Bei diesen Markteintrittsstrategien steht die Balance zwischen Standardisierung und Lokalisierung an erster Linie.

\footnotetext{
${ }^{71}$ Vgl. Hill 2013, S. 435 ff.

${ }^{72}$ Vgl. Belz \& Reinhold 1999, S. 98.

${ }^{73}$ Vgl. Kutschker \& Schmid 2005, S. 820 ff.

${ }^{74}$ Vgl. Hill 2013, S. 439.
} 


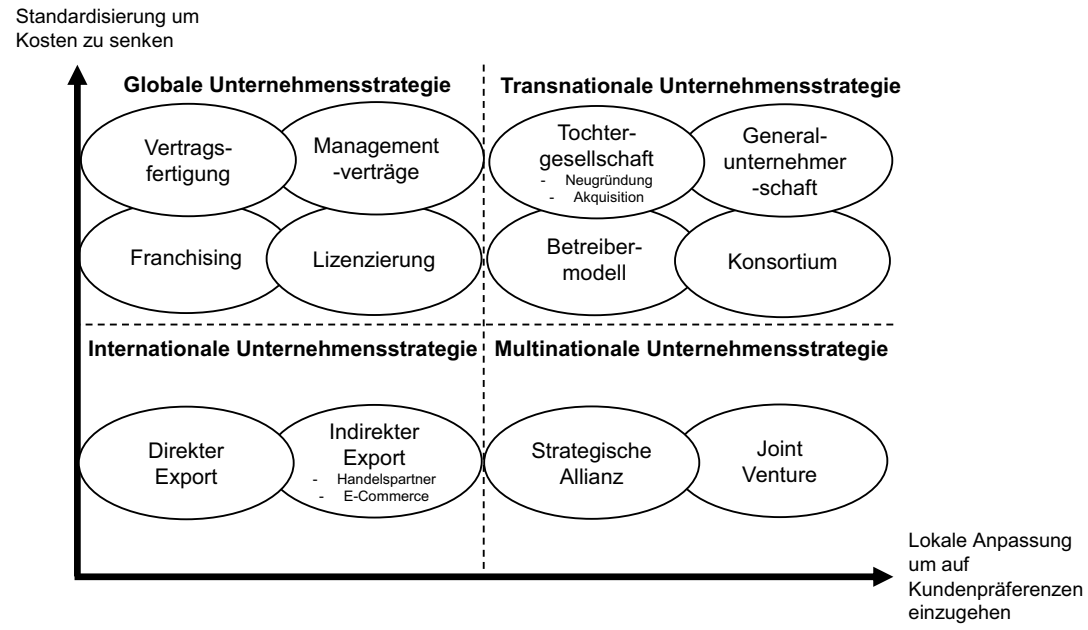

Abb. 2.11 Übersicht über Markteintrittsstrategien je nach Unternehmensstrategie. (Quelle: Eigene Darstellung nach Belz \& Reinhold 1999, S. 98, Hill 2014, S. 398, Holtbrügge \& Welge 2015, S. 107 und Kutschker \& Schmid 2005, S. 820 ff.)

Ein weiteres Modell zur Auswahl von Markteintrittsstrategien ist z. B. die eklektische Theorie der internationalen Produktion von Dunning ${ }^{75}$. Nach Dunning sollten Unternehmen verschiedene Vorteilskategorien überprüfen, z. B. sollten Unternehmen nur dann eine Internationalisierung anstreben, wenn sie mindestens einen Eigentumsvorteil aufweisen können. Im besten Fall ist dieser Eigentumsvorteil mit einem Patent geschützt. Kann ein Unternehmen ein Eigentumsvorteil aufweisen, empfiehlt Dunning einen Markteintritt über Exporte. Weiterhin können Unternehmen Ausschau halten nach Standortvorteilen, z. B. locken Schwellenländer mit niedrigen Lohnkosten Unternehmen aus Industrieländern an. Kann ein Unternehmen sowohl einen Eigentumsvorteil als auch einen Standortvorteil realisieren, bietet sich eine Lizenzierung-Markteintrittsstrategie an. Hat ein Unternehmen die Möglichkeit z. B. entlang der Wertschöpfungskette Unternehmen aufzukaufen oder die Möglichkeit einen wichtigen Schritt in der Wertschöpfungskette selbst zu entwickeln, spricht Dunning von Internalisierungsvorteilen. Bei drei vorweisbaren Vorteilen, sollte ein Unternehmen

${ }^{75}$ Vgl. Dunning 1988, S. 183 ff. 
ausländische Direktinvestitionen in Betracht ziehen (siehe Tab. 2.4). Ausländische Direktinvestitionen sind z. B. eine eigene Vertriebsniederlassung oder eine eigene Produktionsniederlassung. ${ }^{76}$

Tab. 2.4 Übersicht über Markteintrittsstrategien je nach Unternehmensvorteil

\begin{tabular}{l|l|l|l}
\hline Markteintrittsstrategie & $\begin{array}{l}\text { Eigentumsvorteil } \\
(\mathbf{O})\end{array}$ & $\begin{array}{l}\text { Standortvorteil } \\
(\mathbf{L})\end{array}$ & $\begin{array}{l}\text { Internalisierungsvorteil } \\
(\mathbf{I})\end{array}$ \\
\hline $\begin{array}{l}\text { Ausländische } \\
\text { Direktinvestitionen }\end{array}$ & $\mathrm{Ja}$ & $\mathrm{Ja}$ & $\mathrm{Ja}$ \\
\hline Lizenzierung & $\mathrm{Ja}$ & $\mathrm{Ja}$ & Nein \\
\hline Export & $\mathrm{Ja}$ & Nein & Nein \\
\hline
\end{tabular}

Quelle: Eigene Darstellung in Anlehnung an Dunning 1988, S. 199.

Weitere wichtige Kernfragen im Schritt 4 sind: "Welche Ziele haben wir für das Zielland für die ersten 2 Jahre?" und "Können wir erste Vereinbarungen mit Schlüsselkunden abschließen". Bei diesen Kernfragen geht es darum erste Informationen zur Taktik, d. h. der potenziellen Umsetzung der ausgewählten Strategie, einzuholen. Außerdem sollte bei der Planung einer Markteintrittsstrategie unbedingt die letzte Kernfrage zu Schritt 4 beachtet werden: "Welche Handelsabkommen sind insb. bei Produktionsniederlassungen zu beachten?" Bevor sich ein Unternehmen für eine Markteintrittsstrategie entscheidet, können sie in Schritt 3 bei den Außenhandelskammern im jeweiligen Land wichtige Informationen zu den Handelsabkommen für das jeweilige Land erhalten. In den Handelsabkommen werden Wertschöpfungsanteile von ausländischen Unternehmen, die im Inland produzieren wollen, festgelegt ${ }^{77}$.

Baustein 5: Fokussierung auf eine bestimmte Region und einen bestimm-

\section{ten Vertriebskanal}

Die im vorherigen Baustein beschriebenen Markteintrittsstrategien können die Fokussierung auf einen bestimmten Vertriebskanal sowie auf eine bestimmte Kundengruppe beeinflussen. Zum Beispiel gilt es bei einem Joint Venture festzulegen,

\footnotetext{
${ }^{76}$ Vgl. Dunning 1988, S. 183 ff.

${ }^{77}$ Vgl. European Commission 2018.
} 
welcher Partner welchen Vertriebskanal zunächst angeht ${ }^{78}$ und bei einem Handelspartner gilt es festzulegen, welche Kundengruppen als erstes angesprochen werden $^{79}$.

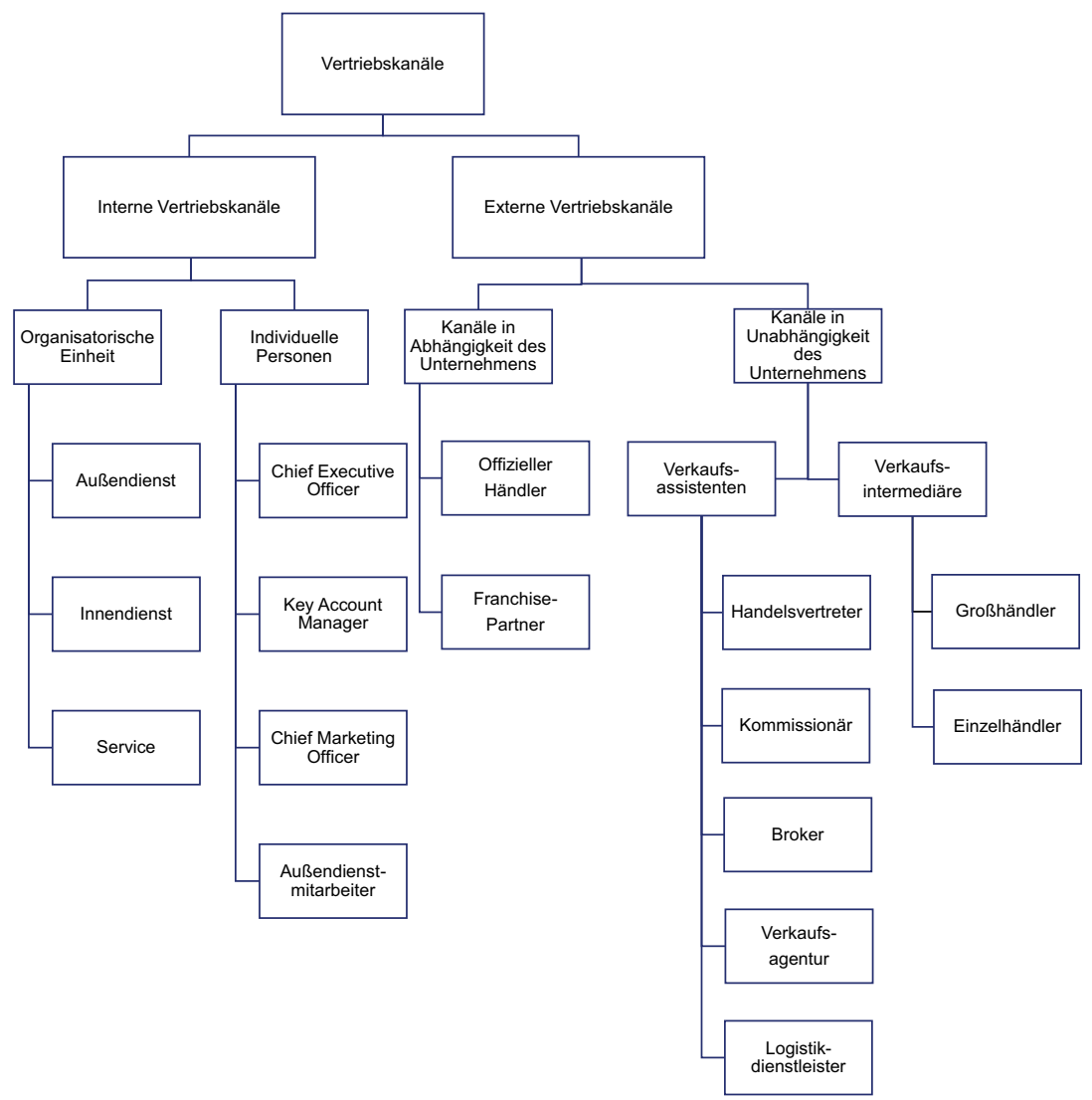

Abb. 2.12 Übersicht über Vertriebsorgane. (Quelle: In Anlehnung an Homburg 2014, S. 257; mit freundlicher Genehmigung von (C) Springer Fachmedien Wiesbaden GmbH 2014. All Rights Reserved)

${ }^{78} \mathrm{Vgl}$. Interview 362017.

${ }^{79}$ Vgl. Interview 312016. 
Dabei ist es wichtig die ganze Auswahl an potenziellen Vertriebskanälen zu kennen. Homburg z. B. unterscheidet zwischen internen und externen Vertriebskanälen (siehe Abb. 2.12) ${ }^{80}$. Bei den internen Vertriebskanälen unterscheidet Homburg zwischen organisatorischen Einheiten und individuellen Personen. Organisatorische Einheiten sind z. B. Außendienst, Innendienst und Service. Individuelle Personen können z. B. der Vorstand, der Key Account Manager, der Marketing Manager oder der Außendienstmitarbeiter sein. Bei den externen Vertriebskanälen können Unternehmen zwischen Kanälen wählen, die abhängig oder unabhängig vom Unternehmen sind. Externe abhängige Vertriebskanäle sind z. B. offizielle Händler oder Franchise-Partner. Bei den externen unabhängigen Vertriebskanälen unterscheidet Homburg zwischen Verkaufsassistenten und Verkaufsintermediären. Verkaufsassistenten können z. B. Handelsvertreter, Kommissionäre, Broker, Verkaufsagenturen oder Logistikdienstleiter sein. Verkaufsintermediäre sind z. B. Großhändler oder Einzelhändler. ${ }^{81}$

Auch zu diesem Schritt gibt es bestimmte Kernfragen, auf die es Antworten zu finden gilt (siehe Abb. 2.13). Eine erste Kernfrage in Schritt 5 lautet: "Welcher Vertriebskanal ist im Zielmarkt besonders wichtig?" Zum Beispiel sind Baumärkte im chinesischen Markt nicht die wichtigsten Vertriebskanäle im Sanitärgeschäft oder Supermärkte sind nicht die wichtigsten Vertriebskanäle für den Verkauf von Teesorten ${ }^{82}$. Die Wichtigkeit von Vertriebskanälen variiert von Land zu Land und muss länderindividuell analysiert werden. Darüber hinaus ist eine weitere Kernfrage in Schritt 5: "In welcher Region können erste Schlüsselkunden akquiriert werden?" Durch die ersten Interviews mit Unternehmen hat sich sehr schnell herauskristallisiert, dass Unternehmen in einer Region mit dem Verkauf ihrer Produkte beginnen. Deshalb bietet sich eine Analyse von potenziellen in Betracht kommenden Regionen an, die je nach Industrie individuell Kriterien beinhalten sollte.

\begin{tabular}{|l|l|l|}
- & Welcher Vertriebskanal ist im Zielmarkt besonders wichtig? \\
- & $\begin{array}{l}\text { In welcher Region können erste Schlüsselkunden akquiriert } \\
\text { werden? }\end{array}$
\end{tabular}

Abb. 2.13 Kernfragen zu Schritt 5. (Quelle: Eigene Darstellung)

\footnotetext{
${ }^{80} \mathrm{Vgl}$. Homburg 2014, S. 257.

${ }^{81}$ Vgl. Homburg 2014, S. 257.

${ }^{82}$ Vgl. Interview 462018 und Interview 432017.
} 
Baustein 6: Erschließung von weiteren Vertriebskanälen und Kundengruppen

In Baustein 6 des International Sales Accelerator Modells geht es um die ErschlieBung von weiteren Vertriebskanälen und Kundengruppen im Auslandsmarkt. Hierbei stellen sich weitere entscheidende Kernfragen (siehe Abb. 2.14). Manager, die für einen Auslandsmarkt zuständig sind, sollten sich zunächst fragen: "Welche weiteren Vertriebskanäle mit möglichst hoher Komplementarität können erschlossen werden?" Diese Frage zielt darauf ab, dem Kunden ein omnipräsentes Vertriebssystem zu schaffen, indem der Kunde jederzeit und überall die Produkte eines Unternehmens kaufen kann.

- Welche weiteren Vertriebskanäle mit möglichst hoher
Komplementarität können erschlossen werden?
- Welche weiteren Kundengruppen können wir erschließen?
- Welche Marketingmaßnahmen können den Bekanntheitsgrad der
eigenen Marke weiter steigern?

Abb. 2.14 Kernfragen zu Schritt 6. (Quelle: Eigene Darstellung)

Laut Gillespie \& Hennessey müssen beim Aufbau von Vertriebskanälen vier Merkmale beachtet werden: Distributionsdichte, Kanalausrichtung, Kanallänge und Distributionslogistik (siehe Tab. 2.5) ${ }^{83}$.

Tab. 2.5 Definition der Merkmale von Vertriebskanälen

\begin{tabular}{l|l}
\hline Merkmale & Definition \\
\hline Distributionsdichte & Marktabdeckung für ein Produkt \\
\hline Kanalausrichtung & Struktur der Kanalmitglieder \\
\hline Kanallänge & Anzahl der Vermittler zwischen Unternehmen und Kunden \\
\hline Distributionslogistik & Physischer Fluss von Produkten
\end{tabular}

Quelle: Eigene Darstellung in Anlehnung an Gillespie \& Hennessey 2016, S. 414.

Dabei verstehen die Autoren unter der Distributionsdichte, die Menge, die zur Abdeckung eines Marktes nötig ist, z. B. die nötige Anzahl von Verkaufsstellen,

${ }^{83}$ Vgl. Gillespie \& Hennessey 2016, S. 414. 
um einen Markt komplett abdecken zu können. Bei der Kanalausrichtung spielt die Struktur der Kanalmitglieder eine große Rolle. Unternehmen müssen sich fragen, ob sie mit der gewählten Struktur eine gemeinsame Strategie erreichen können (siehe Kernfrage 1 zu Schritt 6). Weiterhin erklären Gillespie \& Hennessey, dass die Kanallänge, d. h. die Anzahl der Vermittler zwischen Unternehmen und Kunden, z. B. direkter oder indirekter Kontakt zu den Kunden beachtet werden muss. Bei der Distributionslogistik wird der tatsächliche physische Fluss von Produkten analysiert. ${ }^{84}$

Homburg et al. beschäftigen sich z. B. mit der Klassifizierung und dem Aufbau von Mehrkanalsystemen (siehe Abb. 2.15). In Abb. 2.15 definieren Homburg et al. Basisformen von Mehrkanalsystemen in Abhängigkeit von der Anzahl von direkten und indirekten Vertriebskanälen. Dabei entstehen sechs unterschiedliche Typen von Mehrkanalsystemen. Die Definition solcher Typen ist wichtig, um ein gemeinsames Vokabular für den Aufbau von Vertriebsstrukturen zu schaffen. Darüber hinaus stellen Homburg et al. in einer Studie fest, dass der beliebteste Typ bei Unternehmen im Business-to-Business Geschäft der multiple Direktvertrieb ist, danach folgen die Typen anbietergeprägtes Multi-Channel und differenzierte Multi-Channel ${ }^{85}$.

Außerdem ist laut Homburg et al. zu entscheiden, mit welchen Vertriebskanälen welche Marktsegmente bedient werden sollen (siehe Abb. 2.16) ${ }^{86}$. Die Ergebnisse können in einer sogenannten Coverage-Matrix festgehalten werden.

Stock \& Lambert beschäftigen sich mit der Entwicklung und der Bestimmung von internationalen Distributionssystemen und -strukturen ${ }^{87}$. Die Autoren identifizieren fünf wichtige Kategorien, die ein optimales Distributionssystem benötigt: Umweltanalyse, strategische Planung, Struktur, operative Planung, Controlling. ${ }^{88}$ $\mathrm{Zu}$ jeder Prozesskategorie listen Stock \& Lambert Fragen auf, die sich Manager in den einzelnen Prozessstufen fragen sollten ${ }^{89}$. Weitere Studien zum Ausbau von Vertriebskanälen in ausländischen Märkten beschäftigen sich mit dem Thema Performance von internationalen Vertriebskanälen: Bello \& Gilliland argumentieren, dass die Überwachung von ausländischen Distributoren einen höheren Effekt auf die Performance hat als die Prozesskontrolle an $\operatorname{sich}^{90}$. Rose \& Shoham finden

\footnotetext{
${ }^{84}$ Vgl. Gillespie \& Hennessey 2016, S. 414.

${ }^{85} \mathrm{Vgl}$. Homburg et al. 2012, S. $54 \mathrm{ff}$.

${ }^{86} \mathrm{Vgl}$. Homburg et al. 2012, S. 59 ff.

${ }^{87}$ Vgl. Stock \& Lambert 1983.

${ }^{88}$ Vgl. Stock \& Lambert 1983, S. 40.

${ }^{89}$ Vgl. Stock \& Lambert 1983, S. 40.

${ }^{90}$ Vgl. Bello \& Gilliland 1997, S. 34.
} 


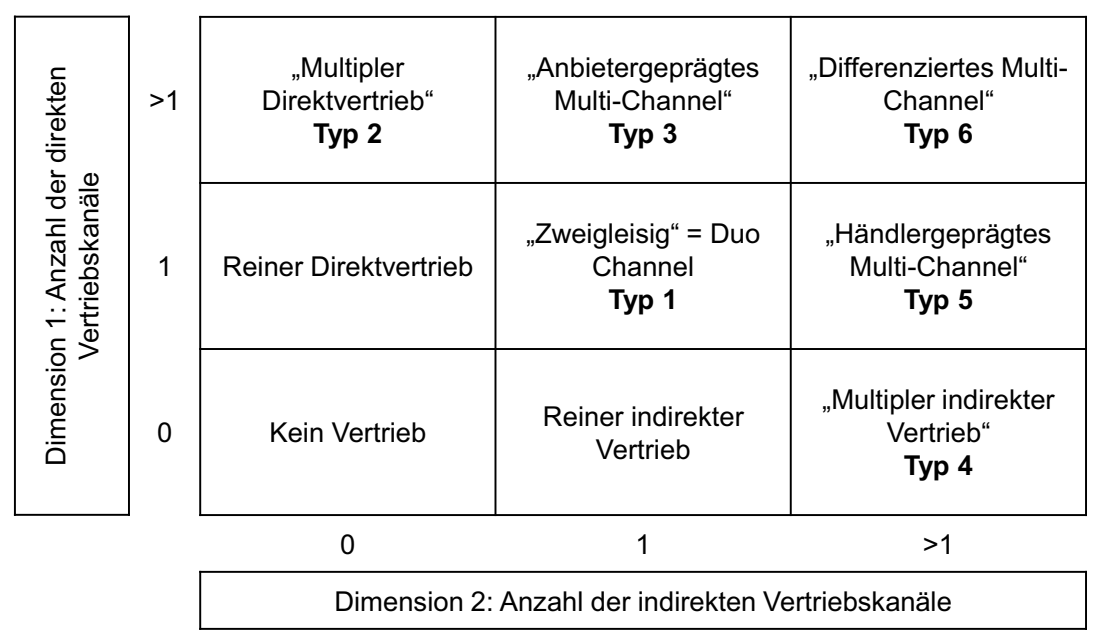

Abb. 2.15 Basisformen von Mehrkanalsystemen. (Quelle: Eigene Darstellung in Anlehnung an Homburg et al. 2012, S. 54; mit freundlicher Genehmigung von (C) Springer Verlag GmbH Deutschland 2012. All Rights Reserved)

dagegen heraus, dass Aufgabenkonflikte und emotionale Konflikte einen negativen Effekt auf die Performance haben ${ }^{91}$. Mehta et al. verifizieren positive Effekte auf das Konstrukt Performance durch die Konstrukte Lernorientierung, Langlebigkeit der Beziehung und Beziehungsnähe ${ }^{92}$. Shoham et al. berichten, dass eine Standardisierung in den internationalen Vertriebskanälen einen positiven Effekt auf die Performance hat ${ }^{93}$. Shoham et al. 's Ergebnisse stehen im Kontrast zu Rosenbloom \& Larsens Ergebnissen. Rosenbloom \& Larsen sind der Auffassung, dass die Vertriebspolitik, die einzige der vier Marketing P's ist, die nicht standardisiert werden sollte $^{94}$. Eine Standardisierung kann sich laut Rosenbloom \& Larsen's Argumentationsweise negativ auf die Performance auswirken. Darüber hinaus finden sie heraus, dass internationale Vertriebskanäle durch gesetzliche Bestimmungen, Marktinfrastruktur, Charakteristika des Marktes sowie den Industriekonditionen bestimmt werden. Nevins \& Money identifizieren, dass das Konstrukt Vertrauen

\footnotetext{
${ }^{91}$ Vgl. Rose \& Shoham 2004, S. 942.

${ }^{92} \mathrm{Vgl}$. Mehta et al. 2006, S. 1094.

${ }^{93}$ Vgl. Shoham et al. 2008, S. 142.

${ }^{94}$ Vgl. Rosenbloom \& Larsen 1991, S. 39.
} 


\begin{tabular}{|c|c|c|c|c|c|c|}
\hline & \multicolumn{6}{|c|}{ Marktsegmente } \\
\hline \multirow{6}{*}{ 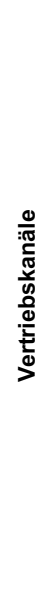 } & & $\begin{array}{l}\text { Behörden / } \\
\text { Öffentliche } \\
\text { Institutionen }\end{array}$ & $\begin{array}{l}\text { Großunterne } \\
\text { hmen }\end{array}$ & Copy-Shops & $\begin{array}{l}\text { Kleine und } \\
\text { mittelständis } \\
\text { che Unter- } \\
\text { nehmen }\end{array}$ & $\begin{array}{l}\text { Sonstige } \\
\text { Abnehmer }\end{array}$ \\
\hline & Außendienst & \multicolumn{2}{|c|}{$\begin{array}{l}\text { Verkauf, Beratung, Wartung, } \\
\text { ggf. Reparatur }\end{array}$} & & & \\
\hline & $\begin{array}{l}\text { Innendienst / } \\
\text { Call Center }\end{array}$ & \multicolumn{5}{|c|}{ Verkauf, Beratung, Hilfestellung bei kleineren technischen Problemen } \\
\hline & Internet & & & \multicolumn{3}{|c|}{ Verkauf, Information } \\
\hline & Großhandel & & & \multicolumn{2}{|c|}{$\begin{array}{l}\text { Lagerhaltung, Verkauf, } \\
\text { Beratung, Wartung, ggf. } \\
\text { Reparatur }\end{array}$} & \\
\hline & $\begin{array}{l}\text { Facheinzel- } \\
\text { handel }\end{array}$ & & & & \multicolumn{2}{|c|}{$\begin{array}{l}\text { Lagerhaltung, Verkauf, } \\
\text { Beratung, Wartung, ggf. } \\
\text { Reparatur }\end{array}$} \\
\hline
\end{tabular}

Abb. 2.16 Coverage-Matrix am Beispiel eines Herstellers von Kopiergeräten. (Quelle: Eigene Darstellung in Anlehnung an Homburg et al. 2012, S. 61; mit freundlicher Genehmigung von (C) Springer Verlag GmbH Deutschland 2012. All Rights Reserved)

einen positiven Effekt auf die Performance eines Unternehmens hat ${ }^{95}$. Weitere Aufsätze zum Thema internationale Vertriebskanäle wurden mit den folgenden Schwerpunkten verfasst: Konfliktmanagement ${ }^{96}$, Distributionssysteme in China ${ }^{97}$, Supply Chain Management ${ }^{98}$, Preispolitik ${ }^{99}$. Ensign z. B. arbeitet an einem Klassifizierungsmodell wie die Forschung von internationalen Vertriebskanälen in Zukunft gegliedert werden $\mathrm{kann}^{100}$. Er unterscheidet strukturelle und verhaltensbezogene Studien, wobei jede Studienart weitere Subkategorien annehmen kann. Strukturelle Studien können einem Entwicklungs-, Transaktionskosten- und vergleichenden Ansatz folgen, während Verhaltensstudien einem relationalen, interkulturellen oder präskriptiven Ansatz nachkommen. ${ }^{101}$

\footnotetext{
${ }^{95}$ Vgl. Nevins \& Money 2008, S. 55.

${ }^{96}$ Vgl. Shoham et al. 2008.

${ }^{97}$ Vgl. Luk 1998 oder Williamson \& Zeng 2004.

${ }^{98}$ Vgl. van Hoek 1998 oder Samiee \& Walters 2006.

${ }^{99}$ Vgl. Vaccaro \& Coward 1993.

${ }^{100}$ Vgl. Ensign 2006, S. 98.

${ }^{101} \mathrm{Vgl}$. Ensign 2006, S. 98.
} 
Eine weitere Kernfrage zu Schritt 6 lautet: "Welche weiteren Kundengruppen können wir erschließen?” Neben dem Ausbau der Vertriebskanäle sollen Kundengruppen definiert und weitere Kundengruppen akquiriert werden. In der Regel beginnen Unternehmen mit den Kundengruppen, die sie aus ihrem Hauptgeschäftsbereich kennen z. B. der Automobilindustrie. Die Produkte und Anlagen sind aber auch für andere Kundengruppen, wie z. B. die Lebensmittelindustrie oder die Chemieindustrie interessant. Wichtig bei dieser Kernfrage ist, einen Plan zu entwerfen welche Industrien relevant sind und wie die Kunden in der jeweiligen Industrie angesprochen werden können. ${ }^{102}$

Eine letzte Kernfrage im Baustein 6 zielt auf weitere Marketingmaßnahmen ab: "Welche Marketingmaßnahmen können den Bekanntheitsgrad der eigenen Marke weiter steigern?". In diesem Punkt geht es um die Einbeziehung der weiteren Marketingpolitiken. Für Konsum- und Industriegüter sind das die Produktpolitik, Kommunikationspolitik und Preispolitik ${ }^{103}$. Bei Dienstleistungen kommen zu den vier Marketingpolitiken noch drei weitere Marketingpolitiken hinzu. Diese sind Ausstattungspolitik, Prozesspolitik und Personalpolitik ${ }^{104}$.

In der akademischen Literatur findet man unter dem Begriff Marktdurchdringungsstrategien nur sehr wenige Studien ${ }^{105}$. Goodnow \& Kosenko z. B. verstehen unter Marktdurchdringungsstrategien vor allem den Ausbau von Vertriebskanälen (siehe Tab. 2.6). Nach der vorliegenden Dissertation sind die in Tab. 2.6 vorgestellten Marktdurchdringungsstrategien Markteintrittsstrategien gemischt mit dem Aufbau erster Vertriebskanäle. Marktdurchdringungsstrategien dagegen sind Strategien wie Unternehmen ihre Marktpräsenz nach und nach in einem ausländischen Markt entwickelten, so dass ihre Produkte und Dienstleistungen dem gesamten Markt zugängig sind (siehe auch nachfolgenden Abschnitt zu eigenen Erkenntnissen aus der Praxis).

Lim et al. argumentieren, dass sogenannte graue Märkte ${ }^{106}$, nicht als Preisproblem, sondern als Markteintrittsstrategie für Start-up Entrepreneure betrachtet werden sollen ${ }^{107}$. Weiterhin sprechen sich Lim et al. dafür aus, dass Start-up

\footnotetext{
${ }^{102} \mathrm{Vgl}$. Interview 12015.

${ }^{103} \mathrm{Vgl}$. Homburg \& Krohmer 2006, S. 557 f.

${ }^{104}$ Vgl. Meffert et al. 2015, S. 268.

${ }^{105}$ Vgl. z. B. Goodnow \& Kosenko 1990, Lim et al. 2001, Meyer \& Thu Tran 2006 oder Millington \& Bayliss 1999.

${ }^{106}$ Graue Märkte treten dann auf, wenn Markenprodukte den Konsumenten durch andere Vertriebskanäle als die vom Hersteller autorisierten Vertriebskanäle erreichen (vgl. Lim et al. 2001, S. 405).
}

${ }^{107}$ Lim et al. 2001, S. 405. 
Tab. 2.6 Marktdurchdringungsstrategien nach Goodnow \& Kosenko

\begin{tabular}{l|l}
\hline Strategie A & Findung einer Nische in einem traditionellen Vertriebskanal \\
\hline Strategie B & Nutzung eines nicht traditionellen Großhändlers \\
\hline Strategie C & $\begin{array}{l}\text { Mitnahme bei einem etablierten japanischen oder ausländischen } \\
\text { Unternehmen }\end{array}$ \\
\hline Strategie D & Zusammenschluss mit einem japanischen Unternehmen \\
\hline Strategie E & Direkter Verkauf an Einzelhändler \\
\hline Strategie F & Direkter Verkauf an Endkunden \\
\hline
\end{tabular}

Quelle: Eigene Darstellung in Anlehnung an Goodnow \& Kosenko 1990, S. 18-20.

Entrepreneure mit Hilfe von grauen Märkten auch ausländische Märkte durchdringen können ${ }^{108}$. Meyer \& Thu Tran analysieren unterschiedliche Markenstrategien bzw. erläutern, dass eine passende Markenstrategie bei der Marktdurchdringung von großer Hilfe sein kann ${ }^{109}$. Millington \& Bayliss sehen dagegen in Joint Ventures eine geeignete Marktdurchdringungsstrategie ${ }^{110}$.

Die Ergebnisse aus der Literaturanalyse zu diesem Baustein zeigen, dass auch dieser Baustein (siehe Baustein 1 und 3) bisher wenig Beachtung in der wissenschaftlichen Literatur erhalten hat. Wichtige Kernfragen zu Baustein 7 (siehe Abb. 2.17) wurden daher aus den eigenen Experteninterviews abgeleitet. Zum Beispiel sollten sich Manager fragen "Welche weiteren Vertriebsstandorte können in dem Zielmarkt ausgewählt werden?" oder "Können wir mit den etablierten Vertriebskanälen die Kunden landesweit bedienen?". Das Ziel in Baustein 7 ist ein nationales Rollout. Dazu sollten sich Manager überlegen "Reicht der Umsatz im Zielmarkt aus, um eine vollwertige Tochtergesellschaft aufzubauen inkl. eigener Beschaffung, Produktion, Forschung \& Entwicklungs-Abteilung, etc.?"

\section{Baustein 7: Rollout im Zielmarkt / Marktdurchdringungsstrategien}

In den nachfolgenden Kapiteln 3 und 4 werden die theoretisch fundierten Kernfragen zu den Bausteinen des ISA-Modells mit empirischen Daten unterfüttert, bevor in Kapitel 5 eine Verknüpfung der theoretischen mit den eigenen empirischen Erkenntnissen erfolgt. Alle ISA-Modell Bausteine in Kapiteln 3 und 4 werden zunächst quantitativ dargestellt in Form einer absoluten Häufigkeitsanalyse einer dichotomen Variablen mit den Ausprägungen (1) Baustein

\footnotetext{
${ }^{108}$ Lim et al. 2001, S. 405.

${ }^{109}$ Vgl. Meyer \& Thu Tran 2006, S. 25.

${ }^{110}$ Vgl. Millington \& Bayliss 1999, S. 635.
} 


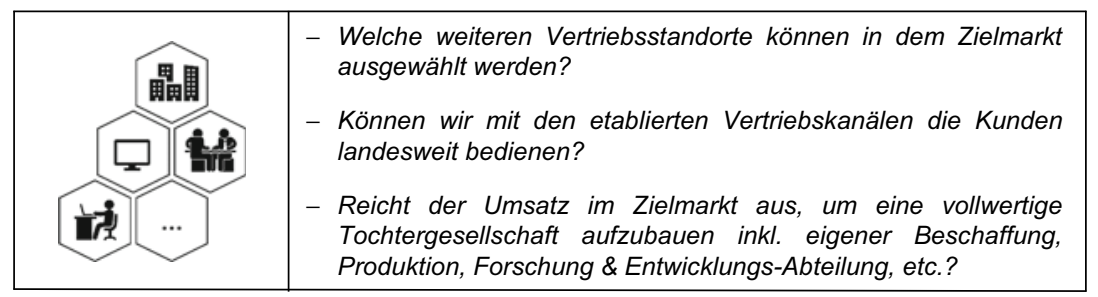

Abb. 2.17 Kernfragen zu Schritt 7. (Quelle: Eigene Darstellung)

wird angewendet und (0) Baustein wird nicht angewendet. Anschließend folgt eine Auswertung der Tätigkeiten zu den einzelnen ISA-Modell Bausteinen. Für die erste Auswertung wurden die Unternehmen anhand ihrer Umsätze (siehe Abschnitt 1.3) in zwei Gruppen, KMUs und GUs, unterteilt. Eine zentrale Annahme hinter dem ISA-Modell ist, dass Großunternehmen im Vergleich zu KMUs mehr ISA-Modell Bausteine anwenden, wenngleich auch die Großunternehmen nicht über alle Tätigkeiten zu den einzelnen Bausteinen verfügen und nur einzelne Tätigkeiten als Module in den Bausteinen bereits umsetzen. ${ }^{111}$

Open Access Dieses Kapitel wird unter der Creative Commons Namensnennung 4.0 International Lizenz (http://creativecommons.org/licenses/by/4.0/deed.de) veröffentlicht, welche die Nutzung, Vervielfältigung, Bearbeitung, Verbreitung und Wiedergabe in jeglichem Medium und Format erlaubt, sofern Sie den/die ursprünglichen Autor(en) und die Quelle ordnungsgemäß nennen, einen Link zur Creative Commons Lizenz beifügen und angeben, ob Änderungen vorgenommen wurden.

Die in diesem Kapitel enthaltenen Bilder und sonstiges Drittmaterial unterliegen ebenfalls der genannten Creative Commons Lizenz, sofern sich aus der Abbildungslegende nichts anderes ergibt. Sofern das betreffende Material nicht unter der genannten Creative Commons Lizenz steht und die betreffende Handlung nicht nach gesetzlichen Vorschriften erlaubt ist, ist für die oben aufgeführten Weiterverwendungen des Materials die Einwilligung des jeweiligen Rechteinhabers einzuholen.

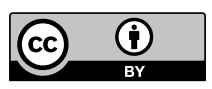

${ }^{111}$ Zur Einteilung der Unternehmen in KMUs und GUs siehe Kapitel 1.3. 